\title{
Chaotic Computation for the Establishment of Unusual Hierarchies of Capability States
}

\author{
Ron Cottam, Roger Vounckx \\ The Living Systems Project, Dept of Electronics and Informatics, Vrije Universiteit \\ Belgium
}

\begin{abstract}
Chaotic computation implies the use of chaotic systems to perform computational operations. Chaotic computation offers a number of interesting possibilities, including the generation of out-of-the-ordinary computational hierarchies of capability states which may mirror those which characterize living systems. In this paper we examine the way in which this may possibly be achieved and note that this kind of hierarchy is inaccessible to computation via formal rationality. Nature appears to rule out the existence of otherthan chaotic highest-performance hierarchies except internally in living systems, and we suggest that this is $a$, or possibly the, primary characteristic of Nature's apparent stability. This, then, also suggests that an approach to chaotic computation may provide a route to reproducing artificially the global characteristics of living systems, including intelligence and consciousness, which are formally unattainable in digital computers.
\end{abstract}

\section{Introduction}

Current artificial information processing is primarily restricted to the domain of Boolean digital computers, although there is some work going on in a rebirth of analog computation [1]. Around the early 21 st century there was evidence of interest in another form of computation, using chaotic systems, but there is no longer much in the way of publication in this area, except in this Journal. The society in which we live has moved rapidly to adopt the primacy of digital approaches to any and all subject areas, to the extent that it is progressively becoming difficult to envisage any other tactic. In this paper we reconsider chaotic computation, but not in such a way as to completely replace Boolean digital computing, which in any case would provide a useful sequential human interface, but specifically as a route towards establishing distributed lifelike processing itself towards simulating the advantages of neural computation in a way which mirrors the capabilities of living systems rather than restricting ourselves to the use of simply reproduced Artificial Neural Networks.

We do not in this paper address the physical implementation of chaotic computation itself, but consider its implications as an extension of the advantages of digital computation. In addition to our direct considerations of chaotic computation we note the possibility that there may be a link between the physical restriction of digital computation caused by the communicational limitation due to the maximal speed of light and the stability of Nature as we know and observe it. We relate the properties of Natural information processing to the tendency of Nature towards hierarchical structures, which we represent by means of a model hierarchy.

\section{Natural hierarchical structure}

Nature tends towards hierarchical structures, although the scavenging character of Evolution [2] means that its results are not always locally hierarchical, as, for example, in some parts of neural processing [3]. However, we should be very circumspect in choosing the particular form of hierarchy which we are referring to. Much information appears in the literature about hierarchical structures, but most of it is referred to business or social contexts [4, 5]. More general approaches applicable to living systems are somewhat sparse $[6,7,8]$ and these concentrate on one of two forms, most characterized by the publications of Stan Salthe [9, 10]. Salthe [10] specifies that, for him, there are only two logically acceptable forms of hierarchy, which he recently refers to respectively as a composition hierarchy and a subsumption hierarchy. The canonical version of the former is a nested set of structural elements, as in the sequence of $\{$ atoms\}, \{molecules\}, \{cells\}, \{organs\}, \{organisms\}, and \{populations\}. The latter is most easily seen as a non-nested sequence of descriptives of a system, as in \{physics\}, \{chemistry\}, \{biology\}. Our work $[11,12]$ concludes that there is at least one more hierarchical structure, namely that of a model hierarchy, which 
has been described by Salthe ${ }^{1}$ as "a subsumption hierarchy constructed in terms of scale". This is no surprise, as a model hierarchy appears to be the parent of Salthe's two formulations, which are both reduced forms of a model hierarchy.

So, what is a model hierarchy? Here, each individual non-nested organizational level of the hierarchy is characterized by a scale-dependent model of the entire system, as in, for example, an organism as atoms\}, \{an organism as molecules $\}$, \{an organism as cells $\}$, up to an organism as itself\}. This last level corresponds to a binary definition of existence.

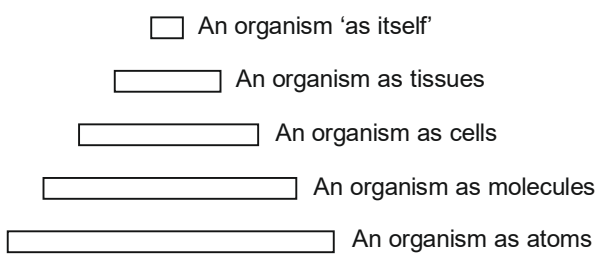

Figure 1. The model-hierarchical structure of an organism

Figure 1 illustrates this hierarchical structure. Each organizational level of the hierarchy is the parent of the next, moving from bottom to top in the figure, which level itself consists of fewer elements or model parameters than its parent (e.g. there are fewer molecules in the system than there are atoms). This has important consequences for transit between adjacent levels, as the level above lacks sufficient information to support movement downwards (in the same manner as in the system $1+1 \rightarrow 2$, where there is a loss of degrees of freedom, and it becomes impossible to know if the left-hand side was originally $1+1$, or $8 / 4$, or...).

There are other extensive and important ramifications of the particular character of a model hierarchy, most particularly when applied to neural processing [13], but these need not concern us directly here. Suffice it to say that we believe that a model hierarchy better represents Nature than any other representation, including both compositional and subsumption hierarchies.

\section{A distinction between info and information}

The word 'information' appears in a multitude of different contexts, with apparently very different meanings. Our reaction to this is to replace the word 'information' by that of 'info' [14], except in the specific case of the ultimate result of neural processing [13]. This eliminates any confusion as to the meaning of 'information' when compared to that

\footnotetext{
${ }^{1}$ Stan Salthe: private communication.
}

of 'info', and gives 'information' a precise sense, while leaving 'info' poorly defined. Information, in its complete instantiation, is then purely subjective, as opposed to conventional definitions such as 'Shannon information' [15], which may well, as info, be objective, but whose character is incomplete in the context of living systems.

In hierarchical processing, organizational levels themselves deal with both info and information as aspects of level emergence in a dual framework between éntity' and 'ecosystem' [14], but the ultimate subjective form of information is singular [14].

\section{Chaotic computation}

Chaotic computation appears mainly in the literature as a route towards improving computer performance or artificial intelligence [16, 17], but its overriding importance is as a component of neural processing itself [18]. Between these two extremes there is the possibility of chaotic computation in artificial neural networks [19], as a model of neural computation itself. The major advantage of chaotic computation has been succinctly expressed by Nicolis [20] as "chaos enables a system to search its phase space". This leads the way to establishing processing which is much faster than a conventional purely formal-logic strategy, and results in a mode of processing which is partway between Classical and Quantum computation. Schroeder [21] has indicated that it is possible to have a system which exhibits a quantum-logical style of operation without itself being a quantum system, and this is the intermediate area in which chaotic computation can operate.

Pribram [22] has proposed that there is a conventionally unnoticed second kind of processing which takes place in neural structures. Conventional approaches to artificial neural networks concentrate solely on weighting effects in the tens of thousands of dendrites at the input of a neuron, to the exclusion of what happens in the axon, or in its extension into a multitude of fine axonites (maybe $\neg 50,000$ ). Pribram has suggested that in the 'axonite mesh' between a (conceptually) first layer of neurons and a (conceptually) second one there is a quasi-quantum wave-nature collapse which transfers info to the secondary neurons. This can be equated to a chaoslike 'computation' between the neurons.

A prime aspect of conventional logic-style processing is the ability to stop its progress in the middle of processing and see 'where it has got to'. This is impossible during the collapse of a quantumlogic system, and also for a chaotic process. The inability to 'stop the process and look how we are doing' halfway through a chaotic computation is equivalent to the impossibility of maintaining quantum system coherent superposition if an observer interacts with the system. 
A major difficulty is to imagine how a useful chaotic computation may be formally instantiated. This is similar in nature to the difficulty, for example, in formally initiating intended oscillation in an electronic oscillator - the start-up of such an effect is due to the presence of low-level noise or switching effects in an unstable context. Consequently, it is difficult to see how to build a 'stand-alone' chaotic processor without specifying completely the context within which it will operate. However, there remains the long-range possibility of establishing chaotic computation in a hierarchical processor - thus reproducing the processing characteristics of a living neural assembly.

\section{The hypothesis: A natural hierarchy of info and information processing}

There is extensive evidence of the use by the brain of hierarchy in its processing of info or information $[23,24,25,26]$. In the same way that we would maintain that our conception of computation is an evolutionarily moulded reduced version of processing in Nature, we hypothesize that the use of hierarchy in the brain is an evolutionarily moulded reduced version of all-encompassing Natural processing structure. We further suggest that its applicability in the brain is uniquely the result of chaotic processing, which enables neural access to the full range of hierarchical organizational levels, such as those of $\mathrm{V} 1, \mathrm{~V} 2, \mathrm{~V} 3, \mathrm{~V} 4 \ldots$ in visual processing [24].

If our hypothesis is correct, then this restriction on formal logical access to higher processing levels except in living systems appears to be of vital importance in establishing the stability of Nature as we know it. We further hypothesize that it may be not only a, but the, most important stabilizing factor. We have earlier published the idea that the Evolution of life itself may be the fundamental nature of evolution in the Universe [13]. This aspect of the limitation of higher-level info or information processing to living systems could be taken to be a supporting argument.

A major question which remains is whether such a multi-levelled processing structure is feasible in an artificially created machine. It is certainly not possible in a conventional Boolean digital computer, where there is no hierarchy at all, and the overriding clock signal segregates all the processing gates from each other. This results in a restriction to local processing and to the absence of any global effects other than those designed-in and uniquely visualized by the computer manufacturer or by its programmer. However, the possibility remains if a processor could be constructed which relies on chaotic computation, rather than the formality of Boolean logic.

\section{A route to emergent unusual multi- levelled processing}

A major limitation of conventional logic-style (Boolean) processing is that it is subject to constraints imposed by the possible physical size of the processing elements, or gates. This becomes a problem in extending the total size of a computer because of the ultimate limitation on maximum communication speed imposed by the speed of light, which restricts the available processing capability in the manner shown in Figure 2.

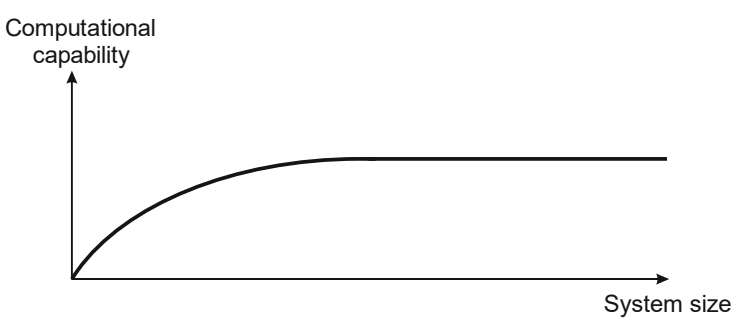

Figure 2. The size-related saturation of computational capability for a physically large system

We believe that neural processing takes place within a model-hierarchical structure [27], but that Boolean logic-style processing always takes place at the lowest organizational level, without any capability to access the next level or levels, as indicated in Figure 3.

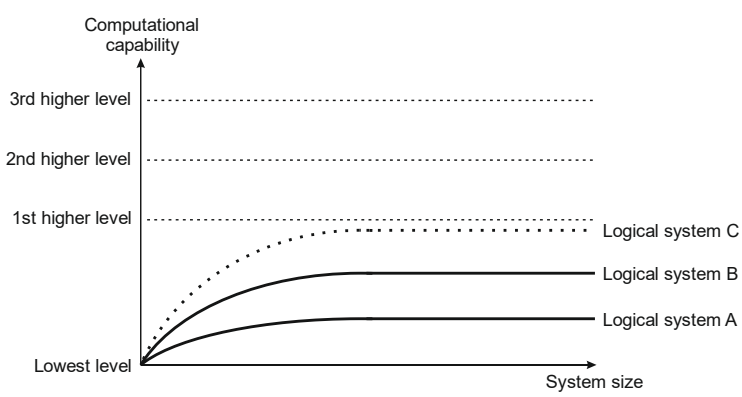

Figure 3. Reducing the processing-element size (logical system A to logical system B) increases the computational capability, but there is a limitation on processing capability imposed by the finite size of atoms which leaves the hypothetical logic system $\mathrm{C}$ still short of being able to access the first higher level of computational capability

Here performance of logical system A levels off beyond a speed-of-light constrained maximum usable size. Reducing the size of the computer's processing elements (logical system B) will increase the computational capability, again as indicated in 
Figure 3, but there is a final restriction in element size (hypothetical logical system C) imposed by the size of the atoms which make up the processing elements. In a more general context than neural processing, we believe that this is a fundamental characteristic of Nature, in that causal logical operations can never achieve access to the higher organizational levels of Natural contexts [28].

The only way of accessing higher organizational levels, then, would be to increase not the total processing capability of a system, but to increase the processing density above that which is achievable using processor-size-dependent elements, such as is the case in a conventional digital computer. This is where chaotic computation comes in, as its feasible processing density is far above that of a conventional computer.



Figure 4. While the three systems A, B and C are restricted in their possible computational capability, hypothetical non-processor-size-dependent chaotic system D is not subject to the same restriction, and

access to the $1^{\text {st }}$ higher level gives it sufficient processing density to then reach the $2^{\text {nd }}$ higher level, and so on

Figure 4 illustrates the comparison between, again, the two capabilities of size-dependent logical processing, $\mathrm{A}$ and $\mathrm{B}$, the hypothetical minimalprocessing-element-size processor $\mathrm{C}$, and the capability of a chaotic processor $\mathrm{D}$, whose processing density is sufficient to enable it to reach the 1st higher available organizational level - it then experiences the emergence of a new, more efficient, processing style. We say 'more efficient' because this next organizational level is a higher-scaled formulation of the computational target, by dint of its reduced elemental or parametric number.

We speculate that this makes the then possible computation sufficient to reach the next highest level, as indicated in Figure 4, and so on to higher and higher levels. Does this characterize, for example, the processing progression between visual cortex levels V1, V2, V3, V4 ... as we have suggested? It is difficult to say, if attractive as a speculation, but the different cortical levels do appear to be formulated as a model hierarchy, with processing at each stage following the edicts of different characterizing models, as in a model hierarchy.

\section{Where to go next?}

Here again, the difficulty of initiating an artificial chaotic computation comes to the fore. Most of the work evidenced in publications envisages using chaotic elements to simulate Boolean gates [16], which is of little interest for the purposes of developing a fundamentally chaotic processor itself. There does not appear to be interest in developing such a processor in the context of a simulation of living systems, although this would be the most valuable goal. Maybe this publication could stimulate such research...

\section{References}

[1] Zuo, S., Wei, Q., Tian, Y., Cheng, Y. and Liu, X. (2018) 'Acoustic analog computing system based on labyrinthine metasurfaces', Scientific Reports 8, p. 10103.

[2] Sigmund, K. (1993) Games of Life: Explorations in Ecology, Evolution, and Behavior, Oxford University Press, New York.

[3] Rossion, B., Dricot, L., Goebel, R. and Busigny, T. (2010) 'Holistic Face Categorization in Higher Order Visual Areas of the Normal and Prosopagnosic Brain: Toward a Non-hierarchical View of Face Perception', Front. Hum. Neurosci. 4, p. 225.

[4] Anderson, C. and Brown, C. E. (2010) 'The functions and disfunctions of hierarchy', Res. Organ. Behav. 27, pp. $1-35$.

[5] Widhiastuti, H. (2012) 'The effectiveness of communications in hierarchical organizational structure', Int. J. Soc. Sci. Human. 2, pp. 185-190.

[6] Salthe, S. N. (1985) Evolving Hierarchical Systems, Columbia University Press, New York.

[7] Pattee, H. H. (1973) Hierarchy Theory - the Challenge of Complex Systems, George Braziller, New York.

[8] Chauvet, G. A. (1993) 'Hierarchical functional organization of formal biological systems: a dynamical approach. I. The increase of complexity by self-association increases the domain of stability of a biological system', Philos. Trans. R. Soc. Lond. B. Biol. Sci. 339, pp. 425-444.

[9] Salthe, S. N. (1993) Development and Evolution: Complexity and Change in Biology, MIT Press, Cambridge.

[10] Salthe, S. N. (2012) 'Hierarchical structures', Axiomathes 22, pp. 355-383. 
[11] Cottam, R., Ranson, W. and Vounckx, R. (2008) 'Toward Artificial Sapience: Principles and Methods for Wise Systems', R.V. Mayorga, and L.I. Perlovsky (eds), Sapient Structures for Intelligent Control', pp. 175-200, Springer, New York.

[12] Cottam, R., Ranson, W. and Vounckx, R. (2008) 'The mind as an evolving anticipative capability', J. Mind Theory 0, pp. 39-97.

[13] Cottam, R. and Ranson, W. (2017) Bridging the Gap between Life and Physics, Springer, Dordrecht.

[14] Cottam, R. and Vounckx, R. (2019) 'Consciousness and Information: Before or After the Event?', submitted to G. Dodig Crnkovic and M. Burgin (eds.) Study of Information, Vol. 2, World Scientific, Singapore.

[15] Shannon, C.E. (1948) 'A mathematical theory of communication', Bell System Technical Journal 27, pp. 379-423.

[16] Munakata, T., Sinha, S. and Ditto, W.L. (2002). 'Chaos computing: Implementation of fundamental logic gates by chaotic elements', IEEE Trans Circuits and Systems - 1. Fundamental Theory and Applications 49 (11), pp. 1629-1633.

[17] Ditto, W.L., Miliotis, A., Murali, K. and Sinha, S. (2010) 'The chaos computing paradigm', in H.G. Schuster (ed.) Reviews of Nonlinear Dynamics and Complexity, pp. 1-35, Wiley Verlag, Weinheim.

[18] Andras, P. (2002) 'Computation with chaotic patterns', Neurocomputing 44-46, pp. 263-268.

[19] Hansel, D. and Sompolinsky, H. (1992). 'Synchronization and computation in a chaotic neural network', Phys. Rev. Letts. 68 (5), pp. 718-721.

[20] Nicolis, G. (1994) Oral presentation in the 2nd European Conference on Artificial Life, VUB UP, Brussels.

[21] Schroeder, M.J. (2009) 'Quantum coherence without quantum mechanics in modeling the unity of consciousness', in Bruza, P., Sofge, D., Lawless, W., van Rijsbergen, C.J. and Klusch, M. (eds.), Quantum Interaction: Proceedings of QI 2009, pp. 97-112, Springer Verlag, Berlin.

[22] Pribram, K.H. (2001) 'Proposal for a quantum physical basis for learning', presented at the 4th International Conference on Emergence, Complexity, Hierarchy and Order, Odense, Denmark, 31 July - 4 August, 2001.

[23] Riesenhuber, M. and Poggio, T. (1999) 'Hierarchical models of objrct recognition in cortex', Nature Neuroscience 2 (11), pp. 1019-1025.

[24] Serre, T. (2012) 'Hierarchical models of the visual system', Nature Reviews Neuroscience 13, pp. 51-62.

[25] Barrett, H.C. (2012) 'A hierarchical model of the evolution of human brain specializations', PNAS 109, pp. 10733-10740.

[26] Jeon, H-A. (2014) 'Hierarchical processing in the prefrontal cortex in a variety of cognitive domains', Frontiers in Systems Neuroscience 8, p. 223.
[27] Cottam, R. and Ranson, W. (2013), 'A biosemiotic view on consciousness derived from system hierarchy', in A. Pereira, Jr and D. Lehmann (eds.), The Unity of Mind, Brain and World, pp. 77-112, Cambridge U P, Cambridge.

[28] Cottam, R., Ranson, W. and Vounckx, R. (1998) 'Emergence: half a quantum jump?', Acta Polytech. Scan. Ma 91, pp. 12-19. 